\title{
A multidisciplinary, home based intervention reduced deaths and readmissions in patients with chronic congestive heart failure
}

\author{
Stewart S, Marley JE, Horowitz JD. Effects of a multidisciplinary, home-based intervention on unplanned readmissions and \\ survival among patients with chronic congestive heart failure: a randomised controlled study. Lancet 1999 Sep \\ 25;354:1077-83.
}

QUESTION: In patients with chronic congestive heart failure (CHF) who are discharged to their homes after acute hospital admission, can a multidisciplinary, home based intervention reduce out of hospital deaths and unplanned readmissions?

\section{Design}

Randomised (allocation concealed*), blinded (outcome assessor),* controlled trial with 6 month follow up.

\section{Setting}

A tertiary referral hospital in Adelaide, South Australia, Australia.

\section{Patients}

200 patients $\geqslant 55$ years of age (mean age $76 \mathrm{y}, 62 \%$ men) who were to be discharged to their homes, had CHF, and had $\geqslant 1$ previous hospital admission for acute CHF. Exclusion criteria were extensive reversible ischaemia that precipitated heart failure, valvular heart disease amenable to surgery, intended heart transplantation, terminal illness, or residence outside of the catchment area. Follow up was complete.

\section{Intervention}

100 patients were allocated to usual care only (appointment with primary care physician or outpatient clinic within $2 \mathrm{wk}$ of discharge and contact with a cardiac rehabilitation nurse, dietitian, social worker, pharmacist, and community nurse, as needed). 100 patients were allocated to usual care and a home based intervention, which included a structured home visit by a cardiac nurse 7 to 14 days after discharge. The nurse sent a report of her findings to the primary care physician and cardiologist. On the basis of the nurse's assessment, patients received remedial counselling and strategies to address areas that needed attention. Home visits were repeated if patients had $\geqslant 2$ unplanned admissions within 6 months. Patients were contacted by telephone at 3 and 6 months.

\section{Main outcome measure}

The primary event was the combined end point of unplanned readmissions and out of hospital deaths.

Source of funding: not stated.

For correspondence: Professor JD Horowitz, Department of Cardiology, Queen Elizabeth Hospital, 28

Woodville Road,

Woodville, South

Australia 5011,

Australia. Fax +618 8222-6030.

\section{Main results}

88 of 100 patients assigned to the home based intervention received a home visit; median duration was 2 hours. At 6 months, the home based intervention group had fewer primary events (unplanned readmissions and out of hospital deaths [77 $v 129, \mathrm{p}=0.02]$ ), fewer unplanned readmissions (68 $v 118, \mathrm{p}=0.03)$, and fewer associated days in the hospital ( $460 v 1174 \mathrm{~d}, \mathrm{p}=0.01$ ) than did the usual care group. The groups did not differ for out of hospital deaths $(9 v 11,\{\mathrm{p}=0.64\} \dagger)$.

\section{Conclusion}

In patients with congestive heart failure who were discharged to their homes, the addition of a multidisciplinary, home based intervention to usual care reduced the rate of unplanned readmissions and the number of days spent in the hospital.

*See glossary.

$\dagger \mathrm{p}$ value calculated from data in article.

\section{COMMENTARY}

Despite the advent of more effective therapy for CHF, the prognosis remains poor. A multidisciplinary approach to CHF that involves therapy, monitoring, education, and counselling may be most efficacious. Stewart and colleagues showed that a multidisciplinary approach that included $\geqslant 1$ home visit by a qualified cardiac nurse was better than usual care that did not include a home visit. These findings are similar to other studies that show a multidisciplinary clinic is more efficacious than usual care. ${ }^{1}$

At the first visit, $40 \%$ of patients had early clinical deterioration, $25 \%$ were not compliant with medical therapy, and $90 \%$ were unaware of the importance of restricting sodium intake. These findings suggest that in-hospital management inadequately prepares the patient and that ongoing comprehensive outpatient management is necessary to maintain long term stability. These hypotheses are further supported by the finding that patients in the intervention group better understood their medications after the home visit. Because approximately $10 \%$ of intervention group patients died or were readmitted before a home visit was done, studies are required to determine a reliable way of timing the first visit after discharge. The availability of several pharmacological therapies increases the complexity of CHF management, further justifying the role of a multidisciplinary programme.

The findings of Stewart and colleagues and others ${ }^{1}$ would support this home based intervention as a cost effective management strategy. Further studies are needed to determine whether this approach is generalisable to smaller, more community based centres; to compare different models of multidisciplinary management; to evaluate the approach over a longer period; and to assess the effects on clinical events in larger, less selected groups of patients.

Robert S McKelvie, $\mathrm{MD}, \mathrm{PhD}$

McMaster University

Hamilton, Ontario, Canada

1 Rich MW. Heart failure disease management: a critical review. J Card Fail 1999;5:64-75. 